\title{
GAMMA-RAY FLARES FROM Mrk421 IN 2008 OBSERVED WITH THE ARGO-YBJ DETECTOR
}

G. Aielli ${ }^{1,2}$, C. Bacci $^{3,4}$, B. Bartoli ${ }^{5,6}$, P. Bernardini $^{7,8}$, X. J. Bi ${ }^{9}$, C. Bleve ${ }^{7,8}$, P. Branchini $^{4}$, A. Budano $^{4}$, S. Bussino $^{3,4}$, A. K. Calabrese Melcarne ${ }^{10}$, P. Camarri ${ }^{1,2}$, Z. CaO $^{9}$, A. Cappa ${ }^{11,12}$, R. Cardarelli $^{2}$, S. Catalanotti ${ }^{5,6}$, C. Cattaneo ${ }^{13}$, P. Celio $^{3,4}$, S. Z. Chen $^{9}$, Y. Chen $^{9}$, N. Cheng ${ }^{9}$, P. Creti ${ }^{8}$, S. W. Cui ${ }^{14}$, B. Z. Dai ${ }^{15}$, G. D’Alí Staiti ${ }^{16,17}$, Danzengluobu ${ }^{18}$, M. Dattoli ${ }^{11,12,19}$, I. De Mitri ${ }^{7,8}$, B. D’Ettorre Piazzoli ${ }^{5,6},{\text { M. De } \text { Vincenzi }^{3,4} \text {, T. Di Girolamo }}^{5,6}$, X. H. Ding $^{18}$, G. Di Sciascio ${ }^{2}$, C. F. Feng ${ }^{20}$, Zhaoyang Feng ${ }^{9}$, Zhenyong Feng $^{21}$, F. Galeazzi ${ }^{4}$, P. Galeotti ${ }^{11,19}$, R. Gargana ${ }^{4}$,

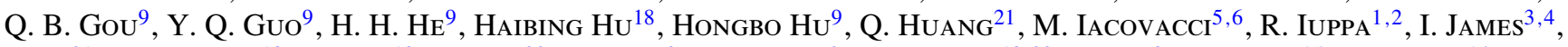

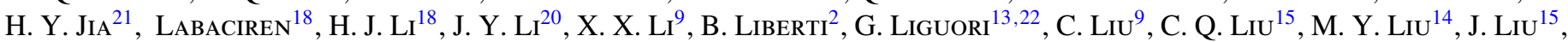

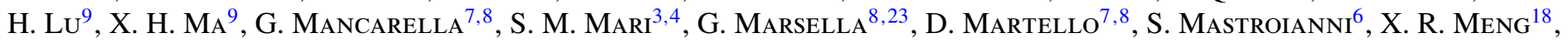
P. Montini ${ }^{3,4}$, C. C. Ning ${ }^{18}$, A. Pagliaro ${ }^{24,16}$, M. Panareo ${ }^{8,23}$, L. Perrone ${ }^{8,23}$, P. Pistilli ${ }^{3,4}$, X. B. Qu $^{20}$, E. Rossi $^{5}$, F. Ruggieri ${ }^{4}$, L. Saggese ${ }^{5,6}$, P. Salvini ${ }^{13}$, R. Santonico ${ }^{1,2}$, P. R. Shen ${ }^{9}$, X. D. Sheng ${ }^{9}$, F. Shi ${ }^{9}$, C. Stanescu ${ }^{4}$, A. Surdo ${ }^{8}$,

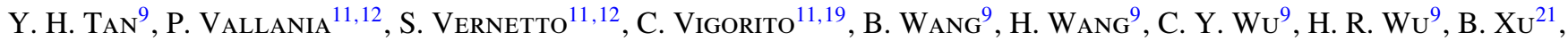

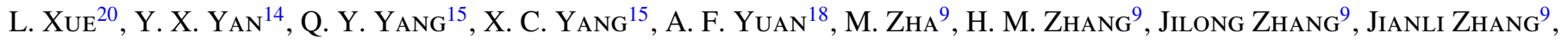

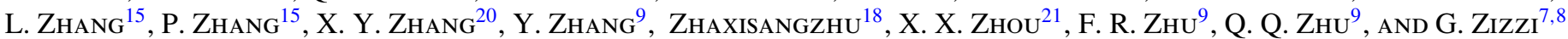

(The Argo-YBJ Collaboration)

${ }^{1}$ Dipartimento di Fisica dell'Università "Tor Vergata" di Roma, via della Ricerca Scientifica 1, 00133 Roma, Italy

2 Istituto Nazionale di Fisica Nucleare, Sezione di Roma Tor Vergata, via della Ricerca Scientifica 1, 00133 Roma, Italy

${ }^{3}$ Dipartimento di Fisica dell'Università "Roma Tre" di Roma, via della Vasca Navale 84, 00146 Roma, Italy

${ }^{4}$ Istituto Nazionale di Fisica Nucleare, Sezione di Roma Tre, via della Vasca Navale 84, 00146 Roma, Italy

${ }^{5}$ Dipartimento di Fisica dell'Università di Napoli, Complesso Universitario di Monte Sant'Angelo, via Cinthia, 80126 Napoli, Italy

${ }^{6}$ Istituto Nazionale di Fisica Nucleare, Sezione di Napoli, Complesso Universitario di Monte Sant’Angelo, via Cinthia, 80126 Napoli, Italy ${ }^{7}$ Dipartimento di Fisica dell'Università del Salento, via Arnesano, 73100 Lecce, Italy

${ }^{8}$ Istituto Nazionale di Fisica Nucleare, Sezione di Lecce, via Arnesano, 73100 Lecce, Italy

${ }^{9}$ Key Laboratory of Particle Astrophysics, Institute of High Energy Physics, Chinese Academy of Science, P.O. Box 918, 100049 Beijing, China

${ }^{10}$ Istituto Nazionale di Fisica Nucleare-CNAF, Viale Berti Pichat 6/2, 40127 Bologna, Italy

${ }^{11}$ Istituto Nazionale di Fisica Nucleare, Sezione di Torino, via P. Giuria 1, 10125 Torino, Italy

12 Istituto di Fisica dello Spazio Interplanetario dell' Istituto Nazionale di Astrofisica, corso Fiume 4, 10133 Torino, Italy

${ }^{13}$ Istituto Nazionale di Fisica Nucleare, Sezione di Pavia, via Bassi 6, 27100 Pavia, Italy

${ }^{14}$ Hebei Normal University, Shijiazhuang 050016, Hebei, China

15 Yunnan University, 2 North Cuihu Road, 650091 Kunming, Yunnan, China

${ }^{16}$ Istituto Nazionale di Fisica Nucleare, Sezione di Catania, Viale A. Doria 6, 95125 Catania, Italy

17 Dipartimento di Fisica e Tecnologie Relative, Università degli Studi di Palermo, Viale delle Scienze, Edificio 18, 90128 Palermo, Italy

18 Tibet University, 850000 Lhasa, Xizang, China

${ }^{19}$ Dipartimento di Fisica Generale dell'Università di Torino, via P. Giuria 1, 10125 Torino, Italy

${ }^{20}$ Shandong University, 250100 Jinan, Shandong, China

${ }^{21}$ South West Jiaotong University, 610031 Chengdu, Sichuan, China

22 Dipartimento di Fisica Nucleare e Teorica dell'Università di Pavia, via Bassi 6, 27100 Pavia, Italy

${ }^{23}$ Dipartimento di Ingegneria dell'Innovazione, Università del Salento, 73100 Lecce, Italy

${ }^{24}$ Istituto di Astrofisica Spaziale e Fisica Cosmica di Palermo, Istituto Nazionale di Astrofisica, Via Ugo La Malfa 153, 90146 Palermo, Italy Received 2009 December 3; accepted 2010 February 24; published 2010 April 13

\section{ABSTRACT}

In 2008, the blazar Markarian 421 entered a very active phase and was one of the brightest sources in the sky at $\mathrm{TeV}$ energies, showing frequent flaring episodes. Using the data of ARGO-YBJ, a full coverage air shower detector located at Yangbajing (4300 m a.s.l., Tibet), we monitored the source at gamma-ray energies $E>0.3 \mathrm{TeV}$ during the whole year. The observed flux was variable, with the strongest flares in March and June, in correlation with X-ray enhanced activity. While during specific episodes the TeV flux could be several times larger than the Crab Nebula one, the average emission from day 41 to 180 was almost twice the Crab level, with an integral flux of (3.6 \pm 0.6$) \times$ $10^{-11}$ photons $\mathrm{cm}^{-2} \mathrm{~s}^{-1}$ for energies $E>1 \mathrm{TeV}$, and decreased afterward. This Letter concentrates on the flares that occurred in the first half of June. This period has been deeply studied from optical to $100 \mathrm{MeV}$ gamma rays, and partially up to $\mathrm{TeV}$ energies, since the moonlight hampered the Cherenkov telescope observations during the most intense part of the emission. Our data complete these observations, with the detection of a signal with a statistical significance of 3.8 standard deviations on June 11-13, corresponding to a gamma-ray flux about 6 times larger than the Crab one above $1 \mathrm{TeV}$. The reconstructed differential spectrum, corrected for the intergalactic absorption, can be represented by a power law with an index $\alpha=-2.1_{-0.5}^{+0.7}$ extending up to several TeV. The spectrum slope is fully consistent with previous observations reporting a correlation between the flux and the spectral index, suggesting that this property is maintained in different epochs and characterizes the source emission processes.

Key words: BL Lacertae objects: individual (Markarian 421) - gamma rays: general

Online-only material: color figures 


\section{INTRODUCTION}

The blazar Markarian 421 has been the first extragalactic source observed at gamma-ray energy $E>500 \mathrm{GeV}$ (Punch et al. 1992). It belongs to the radio-loud active galactic nuclei (AGNs) subclass of BL Lacertae objects, characterized by a non-thermal spectrum extending up to high energies and by a rapid flux variability at nearly all wavelengths.

To date, about 30 BL Lacertae objects have been detected at very high energies (VHE; $E>100 \mathrm{GeV}$ ) and Mrk421 is the closest one $(z=0.031)$. Its relatively small distance makes it one of the best-studied $\mathrm{TeV}$ gamma-ray sources. Since its discovery, this object played a significant role in the discussion concerning both the emission processes in AGNs and the attenuation of $\mathrm{TeV}$ gamma rays in the extragalactic space.

It is now widely recognized that the BL Lacertae object radiation originates in a relativistic jet pointing at a small angle to the line of sight and that it is amplified by relativistic effects, explaining both the strong high-energy emission and its rapid variability.

Usually the BL Lacertae objects' energy density spectra have two broadband components, the first one peaking in the infrared to X-ray region, the second one in the $\mathrm{MeV}-\mathrm{TeV}$ range (Sambruna et al. 1996; Fossati et al. 1998). Mrk421 is classified as a High-energy-peaked BL Lacertae object (HBL), showing the peaks in the X-ray and VHE regions, respectively (Padovani \& Giommi 1995).

The low-energy component is commonly believed to originate as synchrotron emission from relativistic electrons gyrating in the magnetic field of the jet plasma, while the origin of the second one is still unclear. Many models propose that gamma rays are produced in inverse Compton scattering of synchrotron (synchrotron self-Compton, SSC) or ambient photons (external Compton, EC) off the same electron population that produces the synchrotron radiation (Ghisellini et al. 1998; Dermer et al. 1992). Alternatively, in the "hadronic" models, gamma rays are emitted as synchrotron radiation of extremely energetic protons, or by secondary particles produced by protons interacting with some target material (Mücke et al. 2003).

Flaring activity of Mrk421 at VHE energies has been observed with variability timescales ranging from minutes to months, and many multiwavelength campaigns have revealed a strong correlation of gamma rays with X-rays, that can be easily interpreted in terms of the SSC model (Fossati et al. 2008; Wagner 2008). In addition, some data have shown significant variations of the $\mathrm{TeV}$ spectrum slope during different activity phases, and an evident correlation between the spectral hardness and the flux intensity (Krennrich et al. 2002).

The simultaneous observation at different wavelengths is of great importance since it may provide unique information about the source properties and the radiation processes.

A set of measurements (Donnarumma et al. 2009) covering 12 decades of energy, from optical to $\mathrm{TeV}$ gamma rays, was performed during the strong flaring activity in the first half of 2008 June by different detectors: WEBT (optical $R$ band), UVOT (UV), RXTE/ASM (soft X-rays), Swift (soft and hard Xrays), AGILE (hard X-rays and gamma rays), and the Cherenkov telescopes VERITAS and MAGIC (VHE gamma rays). These data allowed for a deep analysis of the broadband energy spectrum as well as for the study of time correlations among the fluxes in different energy ranges.

In this period, two flaring episodes were reported, the first one on June $4-8$, observed from optical to $\mathrm{TeV}$ gamma rays, the second one, larger and harder, on June 10-14, observed from optical to $100 \mathrm{MeV}$ gamma rays. Using the multifrequency data, Donnarumma et al. (2009) derived the spectral energy distribution (SED) for June 6, that shows the typical two humps shape. In the framework of the SSC model, according to the authors, the second hump intensity, which reached a flux of about $3 \times 10^{-11}$ photons $\mathrm{cm}^{-2} \mathrm{~s}^{-1}$ at energies $E>400 \mathrm{GeV}$ (i.e., about 3.5 times the Crab Nebula emission in the same energy range), seems to indicate that the variability is due to the hardening/softening of the electron spectrum, and not due to the increase/decrease of the electron density. Their model predicts the inverse Compton hump slightly shifted toward higher energies for the second flare and a VHE flux, a factor $\geqslant 2$ larger, with respect to the first one.

Unfortunately, there were no VHE data included in their multiwavelength analysis after June 8 because the moonlight hampered the Cherenkov telescopes measurements. The VHE observation was actually made for such a very important flaring episode by the ARGO-YBJ experiment.

The ARGO-YBJ experiment, located at the Yangbajing Cosmic Ray Laboratory (Tibet, $4300 \mathrm{~m}$ a.s.l., $30^{\circ} 06^{\prime} 38^{\prime \prime} \mathrm{N}$, $90^{\circ} 31^{\prime} 50^{\prime \prime} \mathrm{E}$ ), since 2007 December is performing a continuous monitoring of the sky in the declination band from $-10^{\circ}$ to $70^{\circ}$.

In this Letter, we present our observation of Mrk421 in flaring state during 2008. After a summary of the data collected during the most active phase (February-June), we focus our discussion on the results obtained for the June flares in the framework of the Donnarumma et al. (2009) findings.

\section{THE ARGO-YBJ EXPERIMENT}

The ARGO-YBJ detector is constituted by a central carpet $\sim 74 \times 78 \mathrm{~m}^{2}$, made of a single layer of Resistive Plate Chambers (RPCs) with $\sim 93 \%$ of active area, surrounded by a partially instrumented $(\sim 20 \%)$ area up to $\sim 100 \times 110 \mathrm{~m}^{2}$. The apparatus has a modular structure, the basic data acquisition element being a cluster $\left(5.7 \times 7.6 \mathrm{~m}^{2}\right)$, made of $12 \mathrm{RPCs}\left(2.8 \times 1.25 \mathrm{~m}^{2}\right)$. Each chamber is read by 80 strips of $6.75 \times 61.8 \mathrm{~cm}^{2}$ (the spatial pixels), logically organized in 10 independent pads of $55.6 \times$ $61.8 \mathrm{~cm}^{2}$ which are individually acquired and represent the time pixels of the detector. The full detector is made of 153 clusters for a total active surface of $\sim 6600 \mathrm{~m}^{2}$ (Aielli et al. 2006).

ARGO-YBJ operates in two independent acquisition modes: the shower mode and the scaler mode (Aielli et al. 2008). In the following, we refer to the data recorded in shower mode. A simple, yet powerful, electronic logic has been implemented to build an inclusive trigger (Aloisio et al. 2004). This logic is based on a time correlation between the pad signals depending on their relative distance. In this way, all the shower events giving a number of fired pads $N_{\text {pad }} \geqslant N_{\text {trig }}$ in the central carpet in a time window of $420 \mathrm{~ns}$ generate the trigger. This trigger can work with high efficiency down to $N_{\text {trig }}=20$, keeping negligible the rate of random coincidences.

The time of each fired pad in a window of $2 \mu$ s around the trigger time and its location are recorded and used to reconstruct the position of the shower core and the arrival direction of the primary particle (Di Sciascio et al. 2008). In order to perform the time calibration of the 18,360 pads, a software method has been developed (Aielli et al. 2009a).

The detector is in stable data taking with the trigger condition $N_{\text {trig }}=20$ and a duty cycle $\geqslant 85 \%$. The trigger rate is $\sim 3.6 \mathrm{kHz}$ with a dead time of $4 \%$. 


\section{DETECTOR PERFORMANCE}

The angular resolution and the pointing accuracy of the detector have been evaluated by using the Moon shadow, i.e., the deficit of cosmic rays in the Moon direction. The shape of the shadow provides a measurement of the detector pointspread function (PSF), and its position allows the individuation of possible pointing biases. ARGO-YBJ observes the Moon shadow with a sensitivity of about 10 standard deviations per month for events with a multiplicity $N_{\text {pad }} \geqslant 40$ and zenith angle $\theta<50^{\circ}$, corresponding to a proton median energy $E_{p} \sim 1.8 \mathrm{TeV}$ (Di Sciascio et al. 2009).

According to the Moon shadow data, the PSF of the detector is Gaussian for $N_{\text {pad }} \geqslant 100$, while for lower multiplicities it can be described with an additional Gaussian, which contributes for about $20 \%$. When the PSF is a Gaussian with rms $\sigma$, the opening angle $\psi$ containing $\sim 71.5 \%$ of the events maximizes the signal to background ratio for a point source with a uniform background, and is equal to $1.58 \sigma$.

The semi-aperture $\psi$ is found to be $2.59 \pm 0.16,1.30 \pm 0.14$, and $1.04 \pm 0.12$ for $N_{\mathrm{pad}} \geqslant 40,100$, and 300, respectively, in agreement with expectations from Monte Carlo simulations.

This measured angular resolution refers to cosmic ray induced air showers. The angular resolution for $\gamma$-induced events has been evaluated by simulations and results smaller by $\sim 30 \%-40 \%$, depending on $N_{\mathrm{pad}}$, due to the better defined time profile of the showers.

The relation between the observed pad/strip multiplicity spectrum and the primary energy spectrum has been studied with cosmic ray showers, by means of a full Monte Carlo simulation, including the CORSIKA code (Heck et al. 1998) to describe the shower development in the atmosphere, and a code based on the GEANT package (Brun \& Carminati 1994) to simulate the detector response. Primary particles have been sampled from the energy spectra of Hörandel (2003). The measured strip multiplicity spectrum is in good agreement with the one predicted by the simulation (Aielli et al. 2009b).

\section{DATA ANALYSIS}

The data set for the analysis of Mrk421 presented in this Letter contains all showers with $N_{\text {pad }} \geqslant 40$ and zenith angle less than $40^{\circ}$. No event selection based on the shower core position and no gamma-hadron discrimination have been applied in this work.

A sky map in celestial coordinates (right ascension and declination) with $0.1 \times 0.1$ bin size, centered on the source location, is filled with the detected events. In order to extract the excess of $\gamma$-rays coming from the source, the cosmic ray background must be carefully estimated and subtracted from the event map.

The background is evaluated with the time-swapping method (Alexandreas et al. 1993). For each detected event, $N$ "fake" events are generated by replacing the original arrival time with new ones, randomly selected from a buffer that spans a time $T$ of data taking. We chose $T \sim 3 \mathrm{hr}$ to minimize the systematic effects due to the environmental parameters variations. Changing the time, the fake events maintain the same declination of the original event, but have a different right ascension. With these events, a new sky map (background map) is built. The number of fake events generated for each event is $N=T(\mathrm{hr}) \times 15 \times \cos (\delta) / 2 \psi$, where $\psi$ is the radius of the observational window in degrees (see below) and $\delta$ is the declination of the source. In this way, the average number of fake events falling in the observational window is $\sim 1$.
The two maps are then "integrated" over a circular area of radius $\psi$, i.e., every bin is filled with the content of all bins whose center has an angular distance less than $\psi$ from its center, with $\psi=1.7,0.9$, and 0.6 for $N_{\text {pad }} \geqslant 40,100$, and 300, respectively.

Finally, the integrated background map is subtracted to the corresponding integrated event map, obtaining the "source map," where for every bin the statistical significance of the excess is calculated.

With this procedure, however, since the source events are also used in the time-swapping procedure, the obtained background at the source position is slightly overestimated, and the signal underestimated. This underestimation increases with the observational window size, ranging from $\sim 4 \%$ to $10 \%$ of the signal, depending on the $N_{\text {pad }}$ interval. The observed event rate is then corrected using the appropriate factor.

The whole procedure has been tested with the Crab Nebula, the standard candle for VHE astronomy. At the Yangbajing latitude, the Crab culminates at a zenith angle $\theta_{\text {culm }}=8.1$ and it is observable every day for $5.8 \mathrm{hr}$ with a zenith angle $\theta<40^{\circ}$.

The Crab Nebula was observed from 2007 December 13 to 2009 August 8, for a total of 3150 on-source hours, obtaining a signal with a statistical significance of 7.6 standard deviations for $N_{\text {pad }} \geqslant 40$. The average number of gamma rays detected per day is $156.6 \pm 20.6$ for $N_{\text {pad }} \geqslant 40$.

To evaluate the energy spectrum, we simulate a source in the sky following the daily path of the Crab Nebula, and estimate the number of events expected in different $N_{\text {pad }}$ intervals, as a function of the spectrum parameters.

Assuming a power law spectrum in the $0.1-80 \mathrm{TeV}$ energy range, the best fit to the data is $d N / d E=(4.1 \pm 0.6) \times 10^{-11}$ $(E / 1 \mathrm{TeV})^{-2.7 \pm 0.2}$ photons $\mathrm{cm}^{-2} \mathrm{~s}^{-1} \mathrm{TeV}^{-1}$, in agreement with our previous measurement (Vernetto et al. 2010) and with observations by other detectors, such as H.E.S.S. (Aharonian et al. 2006), MAGIC (Albert et al. 2008), and Tibet AS- $\gamma$ (Amenomori et al. 2009). This result confirms the reliability of the simulation procedure and of the energy calibration of the detector.

Concerning the energy range sampled in the Crab Nebula measurement, about $84 \%$ of the detected events comes from primary photons of energies greater than $300 \mathrm{GeV}$, while only $8 \%$ comes from primaries above $10 \mathrm{TeV}$.

The same analysis was performed for Mrk421. This source culminates at the ARGO-YBJ location at a zenith angle $\theta_{\text {culm }}=$ 8.1 , and it is observable every day for $6.4 \mathrm{hr}$ with a zenith angle $\theta<40^{\circ}$.

We evaluate the Mrk421 spectrum from day 41 to 180 of 2008, when the X-ray flux showed the most intense flares. In this period (754 observation hours), the signal has a statistical significance of 5.8 standard deviations.

We assume a power law spectrum multiplied by an exponential factor $\mathrm{e}^{-\tau(E)}$ to take into account the absorption of gamma rays in the extragalactic background light (EBL), with the values of the optical depth $\tau(E)$ given by Raue \& Mazin (2008).

The best fit spectrum obtained is $d N / d E=(3.0 \pm 0.5) \times$ $10^{-11}(E / 1.5 \mathrm{TeV})^{-2.4 \pm 0.3} \mathrm{e}^{-\tau(E)}$ photons $\mathrm{cm}^{-2} \mathrm{~s}^{-1} \mathrm{TeV}^{-1}$. The integral flux above $1 \mathrm{TeV}$ is $(3.6 \pm 0.6) \times 10^{-11}$ photons $\mathrm{cm}^{-2}$ $\mathrm{s}^{-1}$, almost twice the Crab Nebula one, i.e., $2.1 \times 10^{-11}$ photons $\mathrm{cm}^{-2} \mathrm{~s}^{-1}$, according to Aharonian et al. (2006).

The values of the spectral index and of the gamma-ray flux averaged over this 140 days period support the correlation between spectral hardness and flux intensity reported by Krennrich et al. (2002) and Albert et al. (2007), based on observations of Mrk421 in different activity states. 

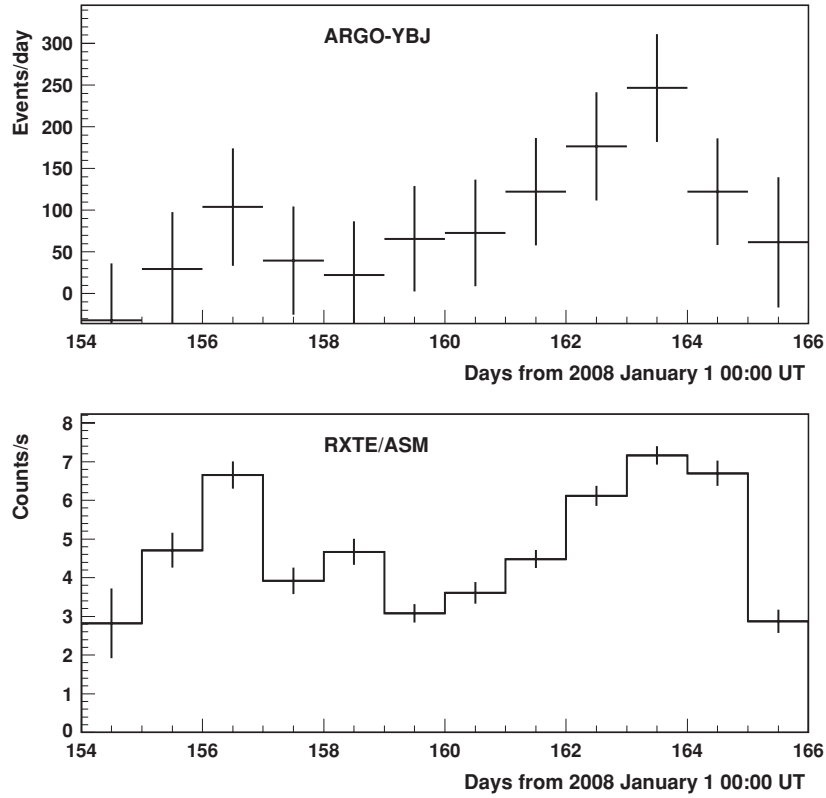

Figure 1. Upper panel: rate of excess events with $N_{\text {pad }} \geqslant 100$ observed from Mrk421 by ARGO-YBJ as a function of time from 2008 June 3 00:00 UT to June 15 00:00 UT. Each bin contains the rate averaged over the 3 days interval centered on that bin. Lower panel: daily counting rate of RXTE/ASM.

A complete account of the observations on Mrk421 will be reported in a dedicated paper.

\section{THE 2008 JUNE FLARES}

As mentioned in the Introduction, two different flares have been observed from Mrk421 in 2008 June, the first one peaking in X-rays on June 4-6 and the second one on June 11-13. Concerning VHE gamma rays, Cherenkov telescopes data are available only for the first flare. An energy spectrum for $E \geqslant 400 \mathrm{GeV}$ has been provided by VERITAS for June 6 .

Since the ARGO-YBJ sensitivity does not allow the observation of a flux a few times larger than the Crab one in only one day (i.e., during one transit of the source in the detector field of view), we integrated the measurement over 3 days.

Figure 1 shows the rate of events with $N_{\text {pad }} \geqslant 100$ observed by ARGO-YBJ from June 3 to June 15, averaged over 3 days, compared with the X-ray flux measured by RXTE/ASM ${ }^{25}$ in the $2-10 \mathrm{keV}$ energy range. A correlation between the gamma-ray and X-ray light curves is clearly visible. During the days June 11-13, when the maximum of the second flare occurred, the excess of events from Mrk421 reached a statistical significance of 3.8 standard deviations.

Beside the statistical error, this measurement could be affected by a systematic uncertainty due to the background evaluation, which is the most delicate step of the analysis. In order to estimate this effect, a completely different procedure for the background calculation has been implemented, using the socalled equi-zenith angle method (Amenomori et al. 2005). In this method, the events collected in 10 off-source windows of the same size of the on-source window, and aligned on both sides of the same zenith angle belt, are used to obtain the background. A detailed study of the two methods in the same sky region has shown that on average they give significances of the excesses consistent within 0.7 standard deviations, corresponding to about $20 \%$ uncertainty on the flux estimate of the observed signal.

\footnotetext{
25 Public quick-look results (http://xte.mit.edu/asmlc/ASM.html).
}

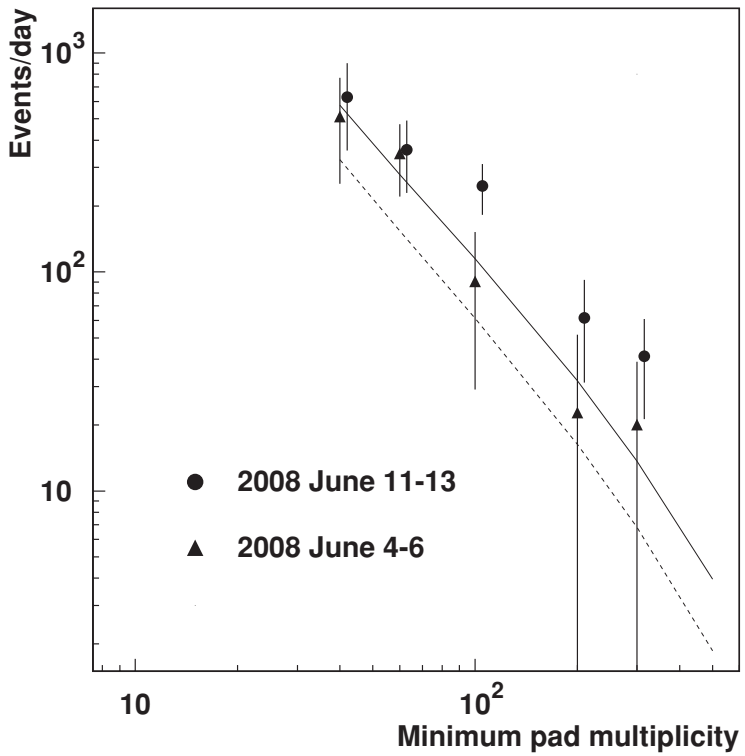

Figure 2. Rates of the excess events observed from Mrk421 by ARGO-YBJ as a function of the event minimum pad multiplicity on 2008 June 4-6 and June 11-13 (triangles and circles, respectively). Expected rates according to the Donnarumma et al. (2009) model for June 6 and June 12-13 (dashed and solid lines, respectively).

(A color version of this figure is available in the online journal.)

The event rate as a function of the minimum pad multiplicity, obtained integrating the data during June 4-6 for the first flare (17.9 hr) and during June 11-13 for the second one (18.2 hr), is shown in Figure 2. On the same plot, the two solid lines represent the expected rates according to the Donnarumma et al. (2009) model, obtained by a simulation procedure. The SED proposed by this model has been corrected for the EBL absorption using the parameters given by Raue \& Mazin (2008) in order to have the flux at Earth. Then, using the absorbed spectrum, we simulated a source moving along the Mrk421 path on the sky, and evaluated the number of events expected in the detector, for different $N_{\text {pad }}$ thresholds. The complete simulation procedure (which includes the gamma ray showers propagation in the atmosphere, and the detector response) has been tested evaluating the Crab Nebula flux, as shown in the previous section.

In the limit of the statistical accuracy of this measurement, our data suggest for both flares a gamma-ray flux higher than that expected by the model, indicating in particular a possible hardening of the spectrum during the second flare.

Considering the first flare, the integral flux measured by ARGO-YBJ above $1 \mathrm{TeV}$ is about 1.5 times higher than the model based on the VERITAS measurement, but still marginally consistent with it. The apparent disagreement between ARGO-YBJ and VERITAS can be likely attributed to the non-coincidence of the data taking periods of the two detectors and to the well-known small variability timescale of the source. The VERITAS data refer to June 6, while the ARGO-YBJ data are integrated over 3 days, from June 4 to 6 . Furthermore, given the difference in longitude of the two detectors $\left(\sim 160^{\circ}\right)$ and the fact that they observe the source during few hours around the culmination time, they can never observe simultaneously the same object.

The disagreement of our data with the model is more significant for the second flare. In order to evaluate the spectral behavior in this period, we assume again a power law spectrum multiplied by the EBL absorption factor $\mathrm{e}^{-\tau(E)}$. From our 


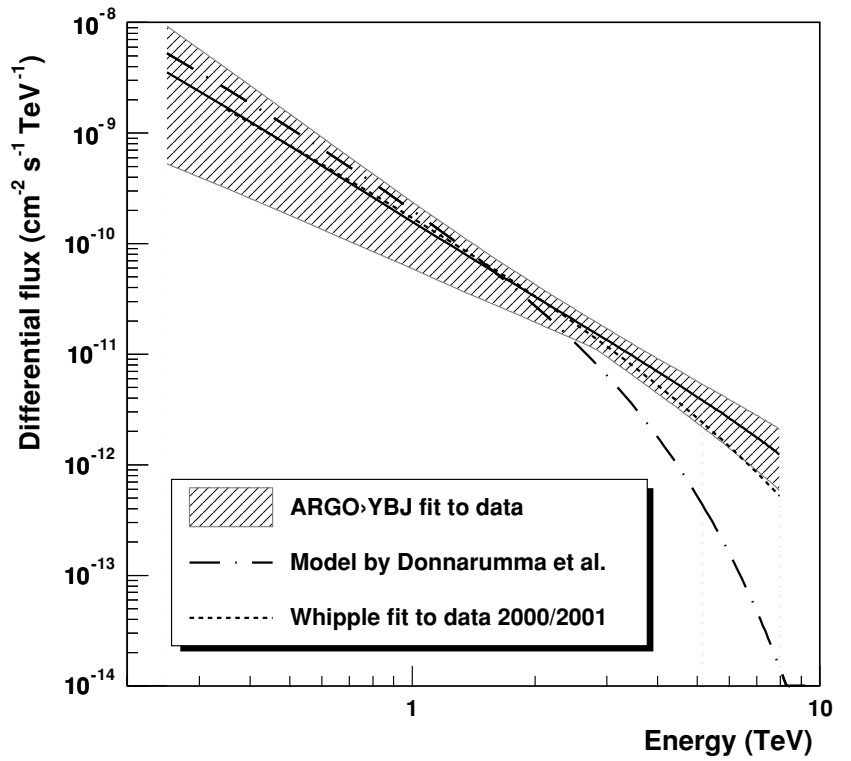

Figure 3. Gamma-ray flux from Mrk421 measured by ARGO-YBJ on 2008 June 11-13 (solid line). The shaded band represents one standard deviation error. The dot-dashed line shows the flux according to the model by Donnarumma et al. (2009) for the second flare (June 12-13). The dotted line shows the spectrum measured by Whipple (Krennrich et al. 2002) during a previous flare of similar intensity (see the text for details).

(A color version of this figure is available in the online journal.)

fitting procedure, we obtain: $d N / d E=(3.2 \pm 1.0) \times 10^{-11}(E /$ $2.5 \mathrm{TeV})^{-2.1_{-0.5}^{+0.7}} \mathrm{e}^{-\tau(E)}$ photons $\mathrm{cm}^{-2} \mathrm{~s}^{-1} \mathrm{TeV}^{-1}$.

This spectrum is shown in Figure 3 as a solid line. The shaded band in the figure represents the $1 \sigma$ statistical error. The systematic errors are mainly related to the background evaluation, as discussed previously, and to the uncertainty in the absolute energy scale. According to our estimate, they globally affect the quoted fluxes for $\lesssim 30 \%$.

Due to the low statistics, our data cannot constrain the shape of the spectrum above $\sim 8 \mathrm{TeV}$. Nevertheless, the obtained flux appears, for energies $>2 \mathrm{TeV}$, significantly larger than predicted by Donnarumma et al. (2009), while the spectrum slope is consistent with that measured by the Whipple Cherenkov telescope during the 2000/2001 observing season for a flare of comparable intensity ( $\sim 7$ times the Crab Nebula flux), also shown in Figure 3 (data set III; Krennrich et al. (2002)).

The integral flux measured above $1 \mathrm{TeV}$ during June 11-13 is $\sim 6$ times larger than the Crab one, making this flare one of the most powerful ever observed from Mrk421.

\section{DISCUSSION AND CONCLUSIONS}

Mrk421 has been continuously monitored by ARGO-YBJ since 2007 December, showing an average VHE flux about twice the Crab Nebula one from 2008 February to June, and decreasing afterward.

Two strong flares in 2008 June have been observed in a multiwavelength campaign from optical to $\mathrm{TeV}$ energies. ARGOYBJ measured the spectra of Mrk421 above $0.3 \mathrm{TeV}$ during the second flare, completing the multifrequency observations. A clear correlation between the gamma-ray intensity measured by ARGO-YBJ and the X-ray flux measured by RXTE/ASM is found.

The ARGO-YBJ data, although averaged over 3 days, appear to support in both episodes a gamma-ray flux higher than that predicted in the analysis of Donnarumma et al. (2009). However, considering the short timescale variability of Mrk421, it has to be noticed that our observation time is not fully coincident with the period referred to by the theoretical curves (June 6 for the first flare and June 12-13 for the second one).

The intensity of the second flare allows us to assess its spectral shape. The deabsorbed spectrum can be fitted by a power law $\propto E^{-2.1_{-0.5}^{+0.7}}$ extending up to several $\mathrm{TeV}$. This spectrum appears definitively harder than that predicted on the basis of June 12-13 data collected up to $\mathrm{GeV}$ energies.

On the contrary, our data follow the behavior of the energy spectra measured during different activity states by the Whipple Cherenkov telescope. In particular, the ARGO-YBJ data fully satisfy the relation between the spectral index and the flux obtained analyzing the measurements of Mrk421 since 1995 (see Figure 3 of Krennrich et al. 2002). This result indicates that this correlation is a long-term property of the source, as previously suggested by the Whipple collaboration.

A global analysis of all the data collected during the 2008 June 11-13 flare, including the present findings, could be used to check the compatibility of the observed phenomenology with current models for VHE photon emission in the jets of AGNs.

We are grateful to the authors of Donnarumma et al. (2009), in particular to Marco Tavani and the AGILE team, for helpful discussions and for providing us the Mrk421 broadband data relative to the period under study.

We also acknowledge the essential supports of W. Y. Chen, G. Yang, X. F. Yuan, C. Y. Zhao, R. Assiro, B. Biondo, S. Bricola, F. Budano, A. Corvaglia, B. D’Aquino, R. Esposito, A. Innocente, A. Mangano, E. Pastori, C. Pinto, E. Reali, F. Taurino, and A. Zerbini, in the installation, debugging, and maintenance of the detector.

\section{REFERENCES}

Aharonian, F., et al. 2006, A\&A, 457, 899

Aielli, G., et al. 2006, Nucl. Instrum. Methods Phys. Res. A, 562, 92

Aielli, G., et al. 2008, Astropart. Phys., 30, 85

Aielli, G., et al. 2009a, Astropart. Phys., 30, 287

Aielli, G., et al. 2009b, Phys. Rev. D, 80, 092004

Albert, J., et al. 2007, ApJ, 663, 125

Albert, J., et al. 2008, ApJ, 674, 1037

Alexandreas, D. E., et al. 1993, Nucl. Instrum. Methods Phys. Res. A, 328 , 570

Aloisio, A., et al. 2004, IEEE Trans. Nucl. Sci., 51, 1835

Amenomori, M., et al. 2005, ApJ, 633, 1005

Amenomori, M., et al. 2009, ApJ, 692, 61

Brun, R., \& Carminati, F. 1994, CERN Program Library Long Writeup, W5013 Dermer, C. D., Schlickeiser, R., \& Mastichiadis, A. 1992, A\&A, 256, L27

Di Sciascio, G., et al. 2008, Proc. 30th ICRC, 4, 123

Di Sciascio, G., et al. 2009, Proc. Vulcano Workshop on Frontier Objects in Astrophysics and Particle Physics Vol. 98, ed. F. Giovannell \& G. Mannocchi (Bologna: SIF), 555

Donnarumma, I., et al. 2009, ApJ, 691, L13

Fossati, G., et al. 1998, MNRAS, 299, 433

Fossati, G., et al. 2008, ApJ, 677, 906

Ghisellini, G., et al. 1998, MNRAS, 301, 451

Heck, D., et al. 1998, Forschungszentrum Karlsruhe Report No., 6019

Hörandel, J. R. 2003, Astropart. Phys., 19, 193

Krennrich, F., et al. 2002, ApJ, 575, L9

Mücke, A., Protheroe, R. J., Engel, R., Rachen, J. P., \& Stanev, T. 2003, Astropart. Phys., 18, 593

Padovani, P., \& Giommi, P. 1995, ApJ, 444, 567

Punch, M., et al. 1992, Nature, 358, 477

Raue, M., \& Mazin, D. 2008, Int. J. Mod. Phys. D, 17, 1515

Sambruna, R. M., Maraschi, L., \& Urry, C. 1996, ApJ, 463, 444

Vernetto, S., et al. 2010, Proc. 2nd Roma Int. Conf. on Astro-Particle Physics, in press

Wagner, R. M. 2008, PoS (BLAZARS2008), 63, 013 (arXiv:0809.2843) 